\title{
RESPOSTAS TERMORREGULADORAS DE CABRAS SAANEN E PARDO ALPINA EM AMBIENTE TROPICAL
}

\author{
THERMOREGULATORY RESPONSES OF SAANEN AND OBERHASLI GOATS \\ INTROPICAL ENVIRONMENTS
}

\author{
Aiura, A.L.O. ${ }^{1 \mathrm{~A}}$, Aiura, F.S. ${ }^{1 \mathrm{~B}}$ e Silva, R.G. ${ }^{2}$ \\ 1'Departamento de Ciências Agrárias. UNIMONTES. Janaúba, MG. Brasil. Aaurilopes@yahoo.com.br; \\ Bfelipe.aiura@unimontes.br \\ ${ }^{2}$ Departamento de Zootecnia. Faculdade de Ciências Agrárias e Veterinárias. UNESP. Jaboticabal, SP. \\ Brasil.robertogs@terra.com.br
}

\section{PaLABRAS ChaVe ADICIONAIS \\ Adaptação. Caprinos. Frequência respiratória. Temperatura retal. Sudação.}

\section{RESUMO}

À forma mais eficaz de termólise quando a carga térmica do ambiente está elevada é a evaporação, uma vez que esse mecanismo não depende do gradiente térmico entre o ambiente $e$ o animal. Deste modo realizou-se observações sobre as resposta termorreguladoras, taxa de sudação (TS), frequência respiratória $(F R)$ e temperatura retal (TR) em 484 cabras das raças Saanen e Pardo Alpina criadas em ambiente tropical. As médias e erros-padrões dessas características foram: TS $=142,91 \pm 4,02 \mathrm{~g} \cdot \mathrm{m}^{-2} \mathrm{~h}^{-1} ; \mathrm{FR}=$ $73,23 \pm 1,60$ mov. $\mathrm{min}^{-1} ; \mathrm{TR}=39,28 \pm 0,02^{\circ} \mathrm{C}$.

\section{SUMMARY}

Evaporation is the most effective way of thermolysis with high environmentmal heat load. This mechanism does not depend on the thermal gradient between the environment and animals. The aim of the present work was thermoregulatory responses, sweating rate (TS), rate frequency (FR) e rectal temperature (TR) of Saanen and Oberhasli goats under tropical environment conditions. The observed averages of responses were: $\mathrm{TS}=142.91 \pm 4.02 \mathrm{~g} \cdot \mathrm{m}^{-2} \mathrm{~h}^{-1} ; \mathrm{FR}=73.23 \pm 1.60$ mov. $\min ^{-1} ; \mathrm{TR}=39.28 \pm 0.02^{\circ} \mathrm{C}$.

\section{INTRODUÇÃO}

Os animais homeotérmicos trocam constantemente calor com o ambiente. Assim, a

\section{AdDitionAL KEYWORDS}

Adaptation. Goats. Respiratory frequency. Rectal temperature. Sweating.

resistência de um animal às altas temperaturas é definida pela sua capacidade em dissipar o calor corporal excessivo, conseguindo manter a sua temperatura interna dentro dos limites da homeotermia.

No ambiente tropical, normalmente a temperatura do ar tende a ser próxima ou maior que a corporal, tornando ineficazes as termólises por condução e convecção. Desse modo o mecanismo de termólise considerado mais eficaz é o evaporativo, por não depender do diferencial de temperatura entre o organismo e a atmosfera (Silva, 1999).

A habilidade de muitos animais para desenvolver-se sob condições quentes baseia-se nas respostas compensatórias, como aumento da temperatura retal e da atividade respiratória. A termólise evaporativa da frequência respiratória (Dmi'el e Robertshaw, 1983, Oliveira et al., 2006) é considerada menos expressiva que a perda do calor cutâneo nos animais a campo, que se torna mais importante nos caprinos, por exporem à radiação uma maior área de superfície em relação à massa do seu corpo que os grandes ungulados (Borut et al., 1979).

O objetivo do trabalho foi estudar a taxa 
de sudação, frequência respiratória e temperatura retal em caprinos das raças Saanen e Pardo Alpina mantidos em uma região tropical.

\section{MATERIAL E MÉTODOS}

As observações foram realizadas em 484 cabras não lactantes das raças Saanen e Pardo Alpina em dois plantéis um situado em Viçosa, MG, Brasil (2045'45" Sul, $42^{\circ} 52^{\prime} 04^{\prime \prime}$ Oeste, $657 \mathrm{~m}$ altitude), onde o clima é do tipo Cwb de Köppen, tropical de altitude, com chuvas durante o verão e temperatura média anual em torno de $19^{\circ} \mathrm{C}$; e o outro em São José do Rio Preto, SP $\left(20^{\circ} 49^{\prime} 11^{\prime \prime}\right.$ Sul, 49²2'46" Oeste, 489 m altitude), onde o clima é do tipo Aw, tropical de altitude, inverno seco e ameno com temperatura média anual de $25,33^{\circ} \mathrm{C}$. As medidas foram realizadas em fevereiro e março, respectivamente, nesses plantéis, que possuíam ambas as raças de animais.

As respostas termorregulatórias mensuradas foram: taxa de sudação $\left(\mathrm{TS}, \mathrm{g} \cdot \mathrm{m}^{-2} \cdot \mathrm{h}^{-1}\right)$, frequência respiratória (FR, mov. $\left.\mathrm{min}^{-1}\right)$ e temperatura retal $\left(\mathrm{TR},{ }^{\circ} \mathrm{C}\right)$.

$\mathrm{O}$ método utilizado para obtenção da taxa de sudação dos animais foi o colorimétrico de Schleger e Turner (1965) na região da paleta, uns $18 \mathrm{~cm}$ abaixo do tronco, onde conforme Das (1995), os caprinos apresentam uma taxa de sudação mais intensa.

A taxa de sudação foi obtida através da fórmula: $\mathrm{S}=(22 \times 3600) / 2,06 \mathrm{t}\left(\mathrm{g} \cdot \mathrm{m}^{-2} \mathrm{~h}^{-1}\right), \mathrm{em}$ que té o tempo médio de virada da coloração dos discos, em segundos. Durante a medição foi ainda observada a frequência respiratória, através dos movimentos do flanco e tomada a temperatura retal, com termômetro digital.

Na ocasião das medições foram anotadas a cada hora as temperaturas do psicrômetro e globo negro (Tgn), além da velocidade do vento $(\mathrm{Vv})$. Para a temperatura radiante média (Trm) e umidade relativa (Ur) foram usadas às fórmulas apresentadas por Silva(2000).
A análise de variância foi realizada pelo método de quadrados mínimos (Silva, 1993) e o teste de Tukey $(\mathrm{p}<0,05)$ para a comparação das médias, utilizando o programa SAS (1998).

O modelo geral para as respostas termorreguladoras foi:

$$
\begin{gathered}
Y_{i j k}=\alpha+L_{i}+R_{j}+S_{i j}+b_{1}\left(V_{i j k}\right)+b_{2}\left(T a_{i j k}\right)+b_{3}\left(U R_{i j k}\right)+ \\
b_{4}\left(T m_{i j k}\right)+b_{5}\left(F R_{i j k}\right)+b_{6}\left(T_{i j k}\right)+b_{7}\left(l_{i j k}\right)+\varepsilon_{i j k l}
\end{gathered}
$$

onde:

$Y_{i j k}$ são a taxa de sudação, a frequência respiratória, a temperatura retal da k-ésima cabra; $L_{i}$ é o efeito fixo do i-ésimo local de coleta $(i=1,2)$; $R$ é o efeito fixo daj-ésimo raça $(j=1,2) ; S_{i j}$ é o efeito fixo da interação do i-ésimo local e j-ésima raça $(k=$ $1,2,3,4) ; b_{1}, b_{2}, b_{3}, b_{4}, b_{5}, b_{6}$ e $b_{7}$ são os coeficientes de regressões sobre a velocidade do vento, a temperatura do ar, a umidade relativa do ar, a temperatura radiante média, a frequência respiratória, a temperatura retal e idade do animal em anos $\varepsilon_{\text {iik }}$ é o resíduo, incluindo o erro aleatório e $\alpha$ é o intercepto.

\section{RESULTADOSEDISCUSSÃO}

As médias das variáveis ambientais encontram-se na tabela $\mathbf{I}$.

Em Viçosa (tabela II) os animais apresentaram uma TS 60\% maior em relação aos animais de São José do Rio Preto, e a raça Pardo Alpina 15\% maior que a Saanen. A FR foi maior nos animais em Viçosa, sendo que os da raça Saanen apresentaram maior média. A interação local e raça para a TR mostrou que os animais de São José do Rio Preto apresentaram maior média, sendo a da raça Saanen maior que a da raça Pardo Alpina.

A média geral da taxa de sudação $(142,91$ $\pm 4,02$ ) foi bem próxima da encontrada por Dmi'el e Robertshaw (1983) de $144 \pm 16$ g. $\mathrm{m}^{-2} \mathrm{~h}^{-1}$ e de Borut et al. (1979), de 142,45 \pm $16,99 \mathrm{~g} \cdot \mathrm{m}^{-2} \mathrm{~h}^{-1}$, ambos em cabras beduínas, que já passaram por anos de seleção natural tornado-as supostamente adaptadas ao clima do deserto. O que nos permite verificar que as cabras do nosso estudo encontraram na sudação uma eficiente via de eliminar o 
TERMORREGULAÇÃO DE CABRAS EM AMBIENTE TROPICAL

Tabela I. Médias, mínimas e máximas das variáveis ambientais por locais de coleta. (Environmental variable mean, minimum and maximum in the collection locations).

\begin{tabular}{lcccc}
\hline \multirow{2}{*}{ Variáveis } & \multicolumn{2}{c}{ São José do Rio Preto } & \multicolumn{2}{c}{ Viçosa } \\
& Média & Min-Max & Média & Min-Max \\
\hline Ta $\left({ }^{\circ} \mathrm{C}\right)$ & 25,49 & $23,00-28,00$ & 28,41 & $22,00-33,00$ \\
Trm $\left({ }^{\circ} \mathrm{K}\right)$ & 291,94 & $280,27-298,94$ & 297,24 & $267,50-326,72$ \\
Tgn $\left({ }^{\circ} \mathrm{C}\right)$ & 24,35 & $21,50-27,00$ & 27,41 & $21,00-33,00$ \\
$\operatorname{Vv}\left(\mathrm{m} . \mathrm{s}^{-2}\right)$ & 0,35 & $0,10-1,10$ & 2,42 & $0,70-9,00$ \\
Ur $(\%)$ & 62,79 & $53,51-76,18$ & 47,59 & $32,36-68,08$ \\
\hline
\end{tabular}

$\mathrm{Ta}=$ Temperatura do ar; Trm= Temperatura radiante media; $\mathrm{Tgn}=$ Temperatura do globo negro; $\mathrm{Vv}=$ Velocidade do vento; Ur= Umidade relativa do ar.

excesso de energia térmica nos trópicos.

A diferença observada na taxa de sudação dos animais entre os locais pode ter sido ocasionada pelas condições edafoclimáticas de cada localidade, corroborado pelos efeitos significativos $(\mathrm{p}<0,01)$ das regressões sobre as variáveis ambientais, velocidade do vendo, temperatura do ar, umidade relativa e temperatura radiante média na análise de variância.

Pela tabela I observamos que Viçosa apresentou maior média de temperatura do ar, o que diminui a troca por calor sensível (convecção e radiação), intensificando a perda por calor latente(Oliveira et al., 2006), que por sua vez é ajudada pela menor umidade e maior movimentação do ar (Silva, 2000).

Os valores das médias da taxa de sudação observadas para as raças, tabela II, foram maiores que a encontrada por Brasil et al. (2000), de 27,45 g.m $\mathrm{m}^{-2} \mathrm{~h}^{-1}$ em cabras Alpinas

Tabela II. Efeito e médias estimadas por quadrado médio das respostas termorregulatórias de caprinos, para local, raça e interação local $x$ raça. (Effect and least squares means of the thermoregulatory responses in goats, to the location, breed and interaction place $\mathrm{x}$ breed).

\begin{tabular}{|c|c|c|c|c|c|}
\hline Parâmetros & Rio Preto ${ }^{1}$ & Viçosa $^{2}$ & Média & CV & Efeito \\
\hline \multicolumn{6}{|c|}{ Taxa de sudação (g.m-2-2-1) } \\
\hline Saanen ${ }^{3}$ & 96,58 & 106,30 & $127,28^{\mathrm{b}}$ & \multirow[t]{3}{*}{11,80} & Local $(\mathrm{L})^{* *}$ \\
\hline Pardo Alpina ${ }^{4}$ & 157,98 & 184,87 & $145,58^{a}$ & & Raça $(R)^{*}$ \\
\hline Média & $101,44^{\mathrm{B}}$ & $171,43^{\mathrm{A}}$ & $142,91 \pm 4,02$ & & $L \times R^{\text {ns }}$ \\
\hline \multicolumn{6}{|c|}{ Frequência respiratória $\left(\mathrm{mov} \cdot \mathrm{min}^{-1}\right)$} \\
\hline Saanen ${ }^{3}$ & $47,63^{\mathrm{Ba}}$ & $96,69^{\mathrm{Aa}}$ & 72,16 & \multirow[t]{3}{*}{7,10} & Local $(L)^{\star *}$ \\
\hline Pardo Alpina ${ }^{4}$ & $47,36^{\mathrm{Ba}}$ & $85,49^{A b}$ & 66,43 & & Raça $(\mathrm{R})^{* *}$ \\
\hline Média & 47,50 & 91,09 & $73,23 \pm 1,60$ & & $L \times R^{* *}$ \\
\hline \multicolumn{6}{|c|}{ Temperatura retal $\left({ }^{\circ} \mathrm{C}\right)$} \\
\hline Saanen ${ }^{3}$ & $39,69^{\mathrm{Aa}}$ & $39,01^{\mathrm{Bb}}$ & 39,35 & \multirow[t]{3}{*}{0,87} & Local $(L)^{* *}$ \\
\hline Pardo Alpina ${ }^{4}$ & $39,44^{\mathrm{Ab}}$ & $39,16^{\mathrm{Aa}}$ & 39,30 & & Raça $(R)^{\text {ns }}$ \\
\hline Média & 39,57 & 39,08 & $39,28 \pm 0,02$ & & $L \times R^{* *}$ \\
\hline
\end{tabular}

$\left.{ }^{*} p<0,05\right) ;{ }^{* *} p<0,01 ; n s:$ Não significativo; ${ }^{n} n=198 ;{ }^{2} n=286 ;{ }^{3} n=238 ;{ }^{4} n=246$.

Médias seguidas da mesma letra, minúscula para coluna e maiúscula para linha, para cada efeito, não diferem estatisticamente pelo teste de Tukey $(p>0,05)$. 


\section{AIURA, AIURA ESILVA}

e por Gayão (1992), 60,08 g.m $\mathrm{m}^{-2} \mathrm{~h}^{-1} \mathrm{em}$ cabritas Saanen, trabalhos realizados sob estresse térmico em câmara climática. Quanto à raça Pardo Alpina ter apresentado maior taxa de sudação pode ser explicado pela maior absorção de calor tanto pelo pelame colorido, como pela pele que é bem pigmentada, estimulando uma maior produção de suor pelas glândulas sudoríparas, já a raça Saanen possui pelame e pele brancos que absorvem menos calor (Silva et al., 2003).

Em Viçosa, os animais apresentaram maior FR, que aumenta com a temperatura do ar (Dmi'el e Robertshaw, 1983, Oliveira et al., 2006). Sendo na raça Saanen maior, possivelmente por utilizar a termólise respiratória com mais ênfase, já que sua taxa de sudação foi menor que a Pardo Alpina. Já a TR das raças Saanen e Pardo Alpina apresentaram-se menores em Viçosa,

\section{BIBLIOGRAFIA}

Borut, A., Dmi'el, R. and Shkolnik, A. 1979. Heat balance of resting and walking goats: comparison of climatic chamber and exposure in the desert. Physiol. Zool., 52: 105-112.

Brasil, L.H.A., Wechesler, F.S., Baccari Junior, F., Gonçalves, H.C. e Bonassi, I.A. 2000. Efeitos do estresse térmico sobre a produção, composição química do leite e respostas termorreguladoras de cabras da raça Alpina. Rev. Bras. Zootecn., 29: 1632-1641.

Das, S.K. 1995. A note on cutaneous evaporative loss in Sirohi goats. Indian Vet. J., 72: 771-772.

Dmi'el, R. and Robertshaw, D. 1983. The control of panting and sweating in the black Bedoin goat: A comparison of two modes of imposing a heat load. Physiol. Zool., 56: 404-411.

Gayão, A.L.B.A. 1992. Efeito do estresse térmico sobre a taxa metabólica e o desempenho produtivo de cabritas Saanen em crescimento. Dissertação (Mestrado em Zootecnia). Faculdade de Medicina Veterinária e Zootecnia. Universidade Estadual Paulista. Botucatu. 68 pp.

Oliveira, A.L., Aiura, F.S., Maia, A.S.C. e Silva, R.G. 2006. Evaporação total em cabras Saanen sob ambientes de sol e a sombra. Em: $43^{\text {a }}$ Reunião provavelmente em decorrência desses animais terem trocado o excedente de calor com o meio, diminuindo assim seu estoque de energia térmica. $O$ animal utiliza a estocagem térmica quando necessário para se termorregular(Oliveira et al., 2007), o que provavelmente aconteceu com os animais de São José do Rio Preto que apresentaram maior média de TR, onde a da raça Saanen foi maior, provavelmente pela menor necessidade de perder calor evidenciada pela menor TS.

\section{AGRADECIMENTOS}

Ao Sérgio Mahfuz, proprietário do Capril Rio Preto e ao Prof. Dr. Marcelo Teixeira Rodrigues, da Universidade Federal de Viçosa pelo apoio ao concederem os animais para o trabalho.

Anual da Sociedade Brasileira de Zootecnia João Pessoa. Anais... João Pessoa, PB.

Oliveira, A.L., Aiura, F.S. e Silva, R.G. 2007.

Estocagem térmica em cabras Saanen sob ambientes de sombra e sol. Em: 44a Reunião Anual da Sociedade Brasileira de Zootecnia. Jaboticabal. Anais... Jaboticabal, SP.

SAS Institute. 1998. SAS/STAT. User's Guide: Statistics. Ver. 6.12. $4^{\text {th }}$ ed. Cary. 842 pp.

Schleger, A.V. and Turner, H.G. 1965. Sweating rates of cattle in the field and their reaction to diurnal and seasonal changes. Aust. J. Agr. Res., 16: 92-106.

Silva, R.G. 1993. Manual de procedimentos em análises por quadrados mínimos. FUNEP. São Paulo. 169 pp.

Silva, R.G. 1999. Estimativa do balanço térmico por radiação em vacas Holandesas expostas ao sol e à sombra em ambiente tropical. Rev. Bras. Zootecn., 28: 1403-1411.

Silva, R.G. 2000. Introdução à bioclimatologia animal. Nobel/FAPESP. São Paulo. 286 pp.

Silva, R.G., Lascala Junior, N. e Tonhati, H. 2003. Radiative properties of the body surface of cattle and other animals. T. ASAE, 46: 913-918.

Archivos de zootecnia vol. 59, núm. 228, p. 608. 